\title{
Statins have immunomodulatory and clinical effects in RA
}

Studies in patients with cardiovascular disease have shown that statins regulate the inflammatory response and affect T-cell subsets; however, evidence for the value of this approach to the treatment of patients with rheumatoid arthritis (RA) is lacking. To test their hypothesis that statins have clinical benefits in this setting, related to their effects on regulatory $\mathrm{T}\left(\mathrm{T}_{\mathrm{REG}}\right)$ cells, Xiang Cheng and colleagues treated 55 patients with RA with a 12-week course of atorvastatin.

Compared with those who did not undergo treatment with the drug, patients with RA who received atorvastatin $20 \mathrm{mg}$ per day showed improved clinical outcomes and reduced serum levels of lipids and inflammatory markers after 12 weeks of therapy: improvements were observed in levels of both LDL and total cholesterol, erythrocyte sedimentation rate, levels of C-reactive protein and 28 -joint disease activity score.
In addition, atorvastatin restored the number and suppressive function of $\mathrm{CD} 24^{+} \mathrm{CD} 25^{+} \mathrm{FOXP}^{+} \mathrm{T}_{\mathrm{REG}}$ cells in vivo, which in both groups of RA patients had been shown in vitro to have compromised function at baseline (in comparison with non-RA controls). "We confirmed that atorvastatin is beneficial for RA patients," reports Cheng, "and the beneficial effects involved the enhancement of $\mathrm{T}_{\mathrm{REG}}$ cells, which could lead to restoration of tolerance."

"Although our study suggests that statin treatment is a promising approach for patients with RA," concludes Cheng, "the routine addition of statins to the RA treatment regimen still calls for evidence from large-scale clinical trials."

Sarah Price

Original article Tang, T. T. et al. Atorvastatin upregulates regulatory $T$ cells and reduces clinical disease activity in patients with rheumatoid arthritis. J. Lipid Res. 52, 1023-1032 (2011) 